\title{
REGULARITY FOR LORENTZ METRICS UNDER CURVATURE BOUNDS
}

\author{
MICHAEL T. ANDERSON
}

\begin{abstract}
Let $(\mathbf{M}, \mathbf{g})$ be an $(n+1)$-dimensional space time, with bounded curvature, with respect to a bounded framing. If ( $\mathbf{M}, \mathbf{g})$ is vacuum, or satisfies a mild condition on the stress-energy tensor, then we show that ( $\mathbf{M}, \mathbf{g})$ locally admits coordinate systems in which the Lorentz metric $\mathbf{g}$ is wellcontrolled in the (space-time) Sobolev space $L^{2, p}$, for any $p<\infty$.
\end{abstract}

\section{INTRODUCTION}

A well-known issue in the geometry of space-times is to understand the regularity of metrics with given bounds on the curvature tensor. This issue arises frequently in discussions and analysis of the behavior at the boundary and definitions of singularities for space-times, c.f. [11], [6], [7], [14] for example.

More specifically, it has been an open problem for some time, cf. [4]-[6], [14] for instance, whether a space-time (M, g) which has curvature bounded in $L^{\infty}$ in a suitable sense has coordinate charts in which the metric $\mathbf{g}=\mathbf{g}_{\alpha \beta}$ is $C^{1, \gamma} \cap L^{2, p}$, for any $\gamma<1, p<\infty$. Here $C^{k, \gamma}$ is the Hölder space of functions whose $k^{\text {th }}$ derivatives are Hölder continuous of order $\gamma$, while $L^{k, p}$ is the Sobolev space of functions with $k$ weak derivatives in $L^{p}$.

The purpose of this paper is to provide an affirmative solution to this problem, at least for vacuum space-times or space-times satisfying a mild condition on the stress-energy tensor.

The solution of the corresponding problem in Riemannian geometry has been known for some time, and it is useful to state the exact result in this context before considering the Lorentzian analogue. Thus, let $(M, g)$ be a Riemannian $n$-manifold, with say $C^{\infty}$ smooth metric $g$. Suppose there exists a point $p \in M$ such that

$$
\operatorname{dist}_{g}(p, \partial M) \geq 1 \text {. }
$$

Let $R=R_{i j k l}$ denote the Riemann curvature tensor of $(M, g)$, and let $B_{p}(r)$ denote the geodesic ball of radius $r$ about $p$ in $(M, g)$. Suppose one has bounds

$$
|R|_{L^{\infty}\left(B_{p}(1)\right)} \leq C, \quad \operatorname{vol}_{g} B_{p}\left(\frac{1}{2}\right) \geq v_{o},
$$

for arbitrary constants $C<\infty, v_{o}>0$. Then there exists a constant $r_{o}>0$, depending only on $C$, $v_{o}$ and $n$, such that the ball $B_{p}\left(r_{o}\right)$ admits a coordinate chart $U=\left\{u_{k}\right\}$, in which the metric $g$ is $C^{1, \gamma} \cap L^{2, p}$, for any $\gamma<1, p<\infty$. Further, there exists a constant $R_{o}$, depending only on $C, v_{o}, n$ and $p$, such that

$$
\left\|g_{i j}\right\|_{L^{2, p}} \leq R_{o}
$$

where the norm is taken over the ball $B_{p}\left(r_{o}\right)$. A proof of this result may be found in [12] for instance. By Sobolev embedding $C^{1, \gamma} \subset L^{2, p}$, for $\gamma=1-\frac{n}{p}$, so that (1.3) also gives a bound on $g_{i j}$ in $C^{1, \gamma}$.

A direct analogue of this result in Lorentzian geometry is false, due to the existence of large families of non-flat space-times for which the curvature norm $|\mathbf{R}|^{2}=\mathbf{R}_{i j k l} \mathbf{R}^{i j k l}$ vanishes identically.

Partially supported by NSF Grant DMS 0072591. 
Thus, consider for instance the class of vacuum plane-fronted gravitational waves on $\mathbb{R}^{4}$, with metric of the form

$$
\begin{gathered}
\mathbf{g}=-d u d v-h(x, y, u) d u^{2}+\left(d x^{2}+d y^{2}\right), \\
\Delta_{(x, y)} h=0 .
\end{gathered}
$$

For such metrics, the two possible scalar invariants in the curvature tensor, namely

$$
|\mathbf{R}|^{2}=\langle\mathbf{R}, \mathbf{R}\rangle=\mathbf{R}_{i j k l} \mathbf{R}^{i j k l} \text {, and }\langle\mathbf{R}, * \mathbf{R}\rangle=\mathbf{R}_{i j k l}(* \mathbf{R})^{i j k l},
$$

vanish identically. The vacuum Einstein equations impose only the condition (1.5), i.e. that $h$ is harmonic as a function of $(x, y)$. Thus, the function $h$ may be an arbitrary function of $u$, and so is not controlled in any Hölder or Sobolev space. It is thus clear that there is no coordinate system in which a general metric $\mathrm{g}$ of the form (1.4) is controlled in $L^{2, p}$, or even $C^{0}$.

To deal with this situation, one imposes bounds on the components of $\mathbf{R}$ in a fixed coordinate system or framing. An efficient way to do this is to choose a future-directed unit time-like vector $T=e_{0}$ and extend it to an orthonormal frame $e_{\alpha}, 0 \leq \alpha \leq n$, where the space-time dimension is $n+1$. Since the space $T^{\perp}$ orthogonal to $T$ is space-like and $O(n)$ is compact, the particular choice of framing for $T^{\perp}$ is unimportant. The norm of $\mathbf{R}$ w.r.t. $T$ is then defined as

$$
|\mathbf{R}|_{T}^{2}=\sum\left(R_{i j k l}\right)^{2}
$$

where the components are w.r.t. the framing $e_{\alpha}$.

Observe that if, at a point $p \in \mathbf{M}$, the vector $T=T_{p}$ is contained in a compact subset $W$ of the future interior null cone $T_{p}^{+} \mathbf{M}$, then the norms (1.6) are all equivalent, with constant depending only on $W$. Hence, if $K$ is a compact subset of the space-time $(\mathbf{M}, \mathbf{g})$ and $T$ is a continuous vector field on $K$, then $T$, (or more precisely, $\operatorname{Im} T$, where $T$ is viewed as a section of the tangent bundle), lies within a compact subset of $T^{+} \mathbf{M}$, where $T^{+} \mathbf{M}$ is the bundle of future interior null cones in the tangent bundle $T \mathbf{M}$.

To state the main result, we need the following definition, which is essentially just a normalization on the size of the region to be considered in ( $\mathbf{M}, \mathbf{g})$, as is (1.1).

Definition 1.1. Let $\Omega$ be a domain in a smooth Lorentz manifold ( $\mathbf{M}, \mathbf{g})$. Then $\Omega$ satisfies the size conditions if the following holds: The domain $\Omega$ admits a smooth time function $t$, with $c_{o}^{-1} \leq\|\nabla t\| \leq c_{o}$, for an arbitrary but fixed constant $c_{o}<\infty$. Further, one has

$$
C_{1}=B_{p}(1) \times[-1,1] \subset \subset \Omega,
$$

i.e. the 1-cylinder $C_{1}$ has compact closure strictly contained in $\Omega$. Here $B_{p}(r)$ is the geodesic $r$-ball about a point $p$ in $S$, where $S=S_{0}=t^{-1}(0)$ and the metric $g$ on $S$ is that induced from $\mathrm{g}$. The product $B_{p}(1) \times[-1,1]$ is identified with a subset of $\Omega$ by the flow of $\nabla t$, i.e. $(q, s) \rightarrow \gamma_{q}(s)$, where $\gamma_{q}(s)$ is the flow line of $\nabla t$, starting at $q$ and terminating on the $s$-level set $S_{s}=t^{-1}(s)$.

Let $T=\nabla t /\|\nabla t\|$ be the corresponding future-directed unit time-like vector field, and set

$$
D=\left.\operatorname{Im} T\right|_{C_{1}} \subset \subset T^{+} \Omega .
$$

The size conditions represent a Lorentzian analogue of the condition (1.1). They can always be realized by choosing $\Omega$ to be a sufficiently small open set in ( $\mathbf{M}, \mathbf{g})$ and rescaling the metric up sufficiently. Essentially, they just serve to normalize the data.

The main result of the paper is then the following:

Theorem 1.2. Let $\Omega$ be a domain in a vacuum $(n+1)$-dimensional space-time $(\mathbf{M}, \mathbf{g}), n \geq 2$, satisfying the size conditions. Suppose that there are constants $C<\infty$ and $v_{o}>0$ such that

$$
|\mathbf{R}|_{T} \leq C, \operatorname{vol}_{g} B_{p}\left(\frac{1}{2}\right) \geq v_{o}
$$


Then there exists a constant $r_{o}>0$, depending only on $C, v_{o}, c_{o}, D$, (and $\left.n\right)$, and a coordinate system $\left(\tau, x_{i}\right), 1 \leq i \leq n$, on the $r_{o}$-cylinder

$$
C_{r_{o}}=D_{p}\left(r_{o}\right) \times\left[-r_{o}, r_{o}\right] \subset C_{1},
$$

such that the components of the metric $\mathbf{g}_{\alpha \beta}$ are in $C^{1, \gamma} \cap L^{2, p}$, for any $\gamma<1, p<\infty$. Here $D_{p}(r)$ is the geodesic $r$-ball about $p$ in the level set $\tau=0$ and the product structure is that induced by the flow of $\nabla \tau$.

Further, there exists a constant $R_{o}<\infty$, depending only on $C, v_{o}, c_{o}, D$ and the exponent $p$, such that, on $C_{r_{o}}$,

$$
\left\|\mathbf{g}_{\alpha \beta}\right\|_{L^{2, p}} \leq R_{o} .
$$

More precisely, for any $k \leq 2$, and $0 \leq \alpha, \beta \leq n$,

$$
\left\|\partial_{\mu}^{k} \mathbf{g}_{\alpha \beta}\right\|_{L_{x}^{2-k, p}} \leq R_{o}
$$

where $\partial_{\mu}^{k}$ denotes any $k$-fold space-time partial derivative and the spatial $L_{x}^{2-k, p}$ norm is taken over any spatial slice $\{\tau=$ const $\}$ in $C_{r_{o}}$. The constant $R_{o}$ is independent of $\tau$ in $\left[-r_{o}, r_{o}\right]$.

The coordinates in Theorem 1.2 are geometrically natural; the time coordinate $\tau$ is a Gaussian (equidistant) coordinate, while the spatial coordinates are chosen to be harmonic on the spatial slices $\{\tau=$ const $\}$. It is not clear if there exist space-time harmonic, i.e wave, coordinates, in which $\mathrm{g}$ has this degree of regularity.

The condition that $(\mathbf{M}, \mathbf{g})$ is vacuum, i.e. $\mathbf{R i c}_{\mathbf{g}}=0$, is used in a rather minor way. It is only used, via the Bianchi identity, to obtain $L^{p}$ bounds on the $2^{\text {nd }}$ time derivatives $\partial_{\tau} \partial_{\tau} \mathbf{g}_{0 \alpha}$ of the components $\mathbf{g}_{0 \alpha}$. This is equivalent to bounds on the $2^{\text {nd }}$ time derivatives on the components of the shift vector of the coordinates, i.e. a bound on the acceleration of the shift. Such acceleration components do not appear in any component of the curvature tensor $\mathbf{R}$. All other estimates on $\partial_{\gamma}^{k} \mathbf{g}_{\alpha \beta}$ are independent of the Einstein equations.

The vacuum condition can be weakened to an assumption on the stress-energy tensor $\mathcal{T}$ in the Einstein equations, of the form

$$
\left\|\nabla_{T} \mathcal{T}\right\|_{L_{x}^{-1, p}} \leq C
$$

Here $L_{x}^{-1, p}$ is the dual space of $L_{o}^{1, q}$, the space of $L_{x}^{1, q}$ functions of compact support on spatial slices $\{\tau=$ const $\}$ within $C_{r_{o}}, p^{-1}+q^{-1}=1$.

The condition (1.13) will be satisfied automatically for many physically natural matter fields, cf. Remark 2.1.

Theorem 1.2 is formulated in such a way that it is easy to pass to limits. Thus, suppose $\left(\mathbf{M}_{i}, \mathbf{g}_{i}\right)$ is a sequence of smooth space-times satisfying the hypotheses of the Theorem. There exist then domains $\Omega_{i} \subset\left(\mathbf{M}_{i}, \mathbf{g}_{i}\right)$, points $p_{i} \in \Omega_{i}$ such that the size conditions (1.7)-(1.8) hold, with $D_{i}$ uniformly compact in $T^{+} \Omega_{i}$, (i.e. $T$ does not become arbitrarily close to null cones). If (1.9) and (1.13) hold uniformly on $\Omega_{i}$, then there is a subsequence which converges to a limit $C^{1, \gamma} \cap L^{2, p}$ space-time ( $\mathbf{M}, \mathbf{g})$, defined at least on an $r_{o}$-cylinder $C_{r_{o}}$. Further, the convergence to the limit is $C^{1, \gamma}$ and weak $L^{2, p}$, and the bound (1.12) holds on the limit.

Define a Lorentz manifold ( $\mathbf{M}, \mathbf{g})$ to be weakly regular if $\mathbf{g}$ is a continuous Lorentz metric, with $\mathbf{g} \in L_{l o c}^{1,2}(\mathbf{M})$. It is well-known, cf. [11], [9] for example, that such metrics have a well-defined curvature tensor $\mathbf{R}$ in the sense of distributions. This leads to the following corollary.

Corollary 1.3. Let (M, g) be a weakly regular Lorentz manifold, and let $\Omega \subset \subset \mathbf{M}$ be a domain with compact closure in $\mathbf{M}$. Suppose the size conditions hold locally on $\Omega$, in that the constant $\frac{1}{2}$ is replaced by a small constant $\delta_{o}$ so that $B_{p}\left(\delta_{o}\right) \subset \subset \mathbf{M}$, for any $p \in \Omega$. Suppose also the bounds (1.9) hold locally and uniformly on $\delta_{o}$ cylinders as in (1.7), centered at any $p \in \Omega$. 
Then $\Omega$ may be covered by a finite atlas of charts in which the metric $\mathbf{g}=\mathbf{g}_{\alpha \beta}$ satisfies all the bounds in (1.12), except for the $L_{x}^{p}$ bound on $\partial_{\tau} \partial_{\tau} \mathbf{g}_{0 \alpha}$. The bounds in (1.12) depend, near $\partial \Omega$, on the distance of $\partial \Omega$ to $\partial \mathbf{M}$.

If in addition the bound (1.13) holds distributionally on $\mathbf{M},($ e.g. ( $\mathbf{M}, \mathbf{g})$ is a weak solution of the vacuum equations), then all bounds in (1.12) hold locally on $(\Omega, \mathbf{g})$.

We refer to the proof of Corollary 1.3 below for the precise meaning that (1.13) holds distributionally.

The proof of Theorem 1.2 and Corollary 1.3 follow in $\S 2$, while $\S 3$ concludes the paper with several remarks and extensions of these results, together with some open problems.

\section{Proofs of the Results.}

In this section, we prove Theorem 1.2 and Corollary 1.3. For clarity, the proof of Theorem 1.2 is divided into several steps, each treating basically separate issues. In the following, as already above, space-time quantities are generally denoted in boldface while spatial quantities are not in boldface.

\section{Step I. (Initial Choice of Domain).}

Let $B_{p}(r)$ be the intrinsic geodesic ball about $p$ in $S$. Since $S$ is achronal in the cylinder $C_{1}$ from (1.7), the extrinsic radius of $B_{p}(r)$ is bounded below for $r$ small. Thus, if $\gamma$ is any space-like curve in ( $\mathbf{M}, \mathbf{g})$ from $p$ to $x \in \partial B_{p}\left(r_{1}\right) \subset S$, for $r_{1}$ small, then the length $L(\gamma)$ satisfies $L(\gamma) \geq l_{o} r_{1}$; the constants $l_{o}$ and $r_{1}$ depend only on $c_{o}$ and $D$ in Definition 1.1.

Let $\mathcal{D}_{r_{1}}$ be the domain of dependence of $B_{p}\left(r_{1}\right)$ in the manifold $(\mathbf{M}, \mathbf{g})$. Thus, by choosing $r_{1}$ sufficiently small, again depending only on $c_{o}, D$, one has

$$
\mathcal{D}_{r_{1}} \subset \subset \Omega,
$$

i.e. $\mathcal{D}_{r_{1}}$ has compact closure in $\Omega$.

The region $\mathcal{D}_{r_{1}}$ is globally hyperbolic and hence any pair of time-related points in $\mathcal{D}_{r_{1}}$ may be joined by a time-like maximizing geodesic in $\mathcal{D}_{r_{1}}$. Recall from Definition 1.1 that the curves $\gamma_{x}$ are the flow lines of $\nabla t$ through $x$. For $r_{2}>0$ small, (to be determined below), let

$$
q=\gamma_{p}\left(-r_{2}\right),
$$

so $q<<p$, i.e. $q$ is to the past of $p$. For $x$ to the future of $p, x>>p$, let

$$
\tau(x)=\operatorname{dist}_{\mathbf{g}}(x, q)-\operatorname{dist}_{\mathbf{g}}(p, q),
$$

so that $\tau(p)=0$ and $\tau(x)>0$, for $x>>p$. The distance $\tau(x)$ is realized by a maximizing time-like geodesic $\sigma_{v}(\tau)=\exp _{q}\left(\tau+\tau_{o}\right) v$ from $q$ to $x$; here $v \in T_{q}^{+} \mathbf{M}$, with $\mathbf{g}(v, v)=-1$, and $\tau_{o}=\operatorname{dist}_{\mathbf{g}}(p, q)$. This normalization gives $p=\sigma_{v_{o}}(0)$, for some $v_{o} \in T_{q}^{+} \mathbf{M}$. Let

$$
N=\nabla \tau
$$

be the corresponding unit time-like vector field, so that $N$ is the tangent vector to geodesics $\sigma$ issueing from $q$. Although $N$ is well-defined and smooth along the individual geodesics $\sigma_{v}(\tau)$, (for any $\tau$ until one reaches the boundary of $(\mathbf{M}, \mathbf{g}))$, at points where the exponential map $\exp _{q}$ has cut or conjugate points, $N$ is not uniquely defined. Of course, past such cut or conjugate points, the geodesics $\sigma_{v}(\tau)$ are no longer maximal. Thus, in general, $\tau$ is merely Lipschitz and $N$, as a vector field, is defined only almost everywhere to the future of $q$. At the end of Step I, (via the work in Step V), it will be seen that in fact $\tau$ and $N$ are smooth, in suitable domains of a definite size. In the following, unless stated otherwise, all geodesics are assumed to be maximal, i.e. they are not continued past the conjugate or cut points of $q$. 
Next, let $\Sigma=\Sigma_{0}=\tau^{-1}(0)$ and similarly let $\Sigma_{\tau}$ be the $\tau$-level set of $\tau$ in $\mathcal{D}_{r_{1}}$. Since the geodesics are maximal, $\sigma_{v}(\tau) \in \Sigma_{\tau}$, and $p \in \Sigma=\Sigma_{0}$. Again, in general, $\Sigma_{\tau}$ is only Lipschitz. For $r_{2} \leq r_{1}$, consider the intrinsic geodesic ball $B_{p}\left(r_{2}\right) \subset S$ and let

$$
\mathcal{C}=\left\{x \in \mathcal{D}_{r_{1}}: x=\sigma(\tau), \tau \leq r_{2}, \text { and } \sigma(\tau) \cap B_{p}\left(r_{2}\right) \neq \emptyset\right\} \subset \mathcal{D}_{r_{1}} .
$$

This is the "cone" of maximal geodesics $\sigma$ starting at $q$, hitting $S$ within $B_{p}\left(r_{2}\right)$, and terminating at time $\tau=r_{2}$.

Observe that the vector field $N$ restricted to $\mathcal{C}$ stays within a compact subset of $T^{+} \mathbf{M}$. In fact, since $N$ is parallel along its geodesic flow lines, this needs to be verified only at the base point $q$, where it holds by construction. It then follows from the curvature bound (1.9) and the remarks following (1.6) that

$$
|\mathbf{R}|_{N} \leq C_{1}=C_{1}(C, D) .
$$

Now the curvature bound (2.5) and the Rauch comparison theorem, cf. [2] for instance, imply that if $r_{2}$ is sufficiently small, depending (explicitly) only on $C_{1}$, then the exponential map $\exp _{q}$ restricted to the interior future null cone in $T_{q}^{+}$, has no conjugate points in $\mathcal{C}$. Thus, $\exp _{q}$ is of maximal rank, and so a local diffeomorphism on $\mathcal{C}$. In fact, for $r_{1}$ is sufficiently small, again depending only on $C$ and $c_{o}$, no time-like or null geodesic within $\mathcal{D}_{r_{1}}$ has conjugate points, and so $\exp _{x}$ is of maximal rank on time-like geodesics in $\mathcal{D}_{r_{1}}, \forall x \in \mathcal{D}_{r_{1}}$.

Since $\mathcal{D}_{r_{1}}$ is globally hyperbolic and without time-like conjugate points, it follows then from [2, Thm.11.16] for instance, that any pair of points $x, y \in \mathcal{D}_{r_{1}}$ with $y>>x$, may be joined by a unique maximizing time-like geodesic in $\mathcal{D}_{r_{1}}$, provided $\mathcal{D}_{r_{1}}$ is future 1-connected, (i.e. any pair of time-like curves joining $x$ and $y$ are homotopic through time-like curves).

In general, $\mathcal{D}_{r_{1}}$ need not be future 1-connected. Consider for example the past null cone of 2-dimensional Minkowski space in hyperbolic coordinates $t \in(-\infty, 0), \phi \in(-\infty, \infty)$,

$$
-d t^{2}+t^{2} d \phi^{2} \text {. }
$$

If $\phi$ is identified periodically, with any period, then the resulting space-time is globally hyperbolic but not future 1-connected. The future exponential map based at any point $q$ has cut points; if the period of $\phi$ is sufficiently small, or if $q$ is sufficiently close to $\{0\}$, then cut points occur arbitrarily close to $q$.

However, $\mathcal{D}_{r_{1}}$ is future 1-connected if it has a simply connected Cauchy surface $S$, i.e. $S \equiv S_{r_{1}}=$ $S \cap \mathcal{D}_{r_{1}}$, for $S$ as in (1.7ff). To see this, let $\gamma_{1}, \gamma_{2}$ be two time-like curves with common endpoints in $\mathcal{D}_{r_{1}}$. The flow of the time-like vector field $\nabla t$ gives a strong deformation retraction of $\gamma_{1} \cup \gamma_{2}$ onto a closed loop $\lambda_{1} \cup \lambda_{2}$ in $S$. If $S$ is simply connected, then $\lambda_{1}$ may be deformed into $\lambda_{2}$ within $S$. These two homotopies, time-like along $\nabla t$ and space-like along the Cauchy surfaces $S_{t}$ may be performed simultaneously, but with the latter at a larger speed than the former, to produce a time-like homotopy from $\gamma_{1}$ to $\gamma_{2}$.

We will prove later that $S$, (or more precisely, a domain in $S$ of a definite size), is simply connected. However, in order not to overburden the arguments to follow with such further issues, we assume in the following, through Step IV, that the Cauchy surface $S \subset \mathcal{D}_{r_{1}}$ is simply connected. This hypothesis will be removed in Step V, using the results obtained in the previous steps.

It follows then that the exponential map $\exp _{q}$ is a diffeomorphism onto $\mathcal{C}$, when restricted to a suitable domain in $T_{q}^{+} \mathbf{M}$. The time function $\tau$ is smooth in $\mathcal{C} \backslash\{q\}$, as are the level sets $\Sigma_{\tau} \cap \mathcal{C}$, and there is a unique maximizing geodesic from $q$ to any point in $\mathcal{C}$.

From now on, we consider $\Sigma_{\tau} \subset \mathcal{C}$, and so let $\Sigma_{\tau}$ denote the prior $\Sigma_{\tau}$ intersected with $\mathcal{C}$. The level sets $\Sigma_{\tau}$ form a foliation of $\mathcal{C}$ by equidistant space-like hypersurfaces, with unit normal $N$.

\section{Step II. (Initial Curvature and Volume Estimates).}


The geodesic congruence $\sigma$ on $(\mathcal{C}, \mathbf{g})$ satisfies the Riccati or transport equation

$$
K^{\prime}+K^{2}+\mathbf{R}_{N}=0
$$

Here $K=D^{2} \tau$ is the $2^{\text {nd }}$ fundamental form or extrinsic curvature of the leaves $\Sigma_{\tau}, \mathbf{R}_{N}$ is the symmetric bilinear form given by $\mathbf{R}_{N}(X)=\langle\mathbf{R}(N, X) X, N\rangle$ and ' is the covariant derivative in the direction $N$. Hence, the bound (2.5) gives

$$
\left|K^{\prime}+K^{2}\right| \leq C_{1} .
$$

This estimate holds on $\Sigma_{\tau}$, for all $\tau \in\left[-r_{2}, r_{2}\right]$. It then follows by standard comparison theory for the Riccati ODE (2.6) that if $r_{2}$ is sufficiently small, depending only on $c_{o}, D$, and $C_{1}$, then

$$
|K|_{L^{\infty}} \leq C_{2}, \quad\left|K^{\prime}\right|_{L^{\infty}} \leq C_{2},
$$

on all $\Sigma_{\tau}, \tau \in\left[-\frac{r_{2}}{2}, r_{2}\right]$. The constant $C_{2}$ depends only on $r_{2}$ and $C_{1}$. The Gauss equation relating the curvature $\mathbf{R}$ of the ambient manifold $(\mathbf{M}, \mathbf{g})$ with that of the spatial slices $\Sigma_{\tau}$ reads

$$
\mathbf{R}_{i j k l}=R_{i j k l}+K_{i k} K_{j l}-K_{i l} K_{j k}
$$

for spatial components $(i j k l)$. This, together with the bounds (2.5) and (2.7), thus gives the bound

$$
\left|R_{g}\right|_{L^{\infty}} \leq C_{3},
$$

on $\Sigma_{\tau}, \tau \in\left[-\frac{r_{2}}{2}, r_{2}\right]$. Let $d K$ be the exterior derivative, (w.r.t. the connection induced by $g$ ), of $K$, when $K$ is viewed as a 1 -form with values in $T \Sigma_{\tau}$, i.e. $d K(X, Y, Z)=\left(\nabla_{X} K\right)(Y, Z)-\left(\nabla_{Y} K\right)(X, Z)$. The Gauss-Codazzi equations are

$$
d K=\mathbf{R}^{N},
$$

where $\mathbf{R}^{N}(X, Y, Z)=\langle\mathbf{R}(N, X), Y, Z\rangle$. Hence, (2.5) also implies

$$
|d K|_{L^{\infty}} \leq C_{3} .
$$

We record also the well-known constraint equations:

$$
\begin{gathered}
\delta K=-d H-\boldsymbol{\operatorname { R i c }}(N), \\
R-|K|^{2}+H^{2}=2 \mathbf{R i c}(N, N)+\mathbf{R},
\end{gathered}
$$

where $H=\operatorname{tr} K$ is the mean curvature and the operators $\delta$ and $d$ are taken on $\Sigma_{\tau}$.

Next, we use the bounds above to obtain a lower volume bound on the spatial slices $\Sigma_{\tau}, \tau \in$ $\left[-\frac{r_{2}}{2}, r_{2}\right]$, from that on the slice $S=S_{0}$ in (1.9). To do this, let $\widehat{S}=S \cap \mathcal{C}$. The domain $\widehat{S}$ may be written as a graph over $\Sigma=\Sigma_{0} \subset \mathcal{C}$ via the time coordinate $\tau$ in the usual way. Thus, each geodesic $\sigma=\sigma_{v}$ intersects $\Sigma$ and $\widehat{S}$ in exactly two points $\sigma\left(\tau_{1}\right), \sigma\left(\tau_{2}\right)$, with $\left|\tau_{i}\right| \leq r_{2}$. For $x=\sigma_{v}\left(\tau_{1}\right) \in \Sigma$, let $u(x)=\tau_{2}-\tau_{1}$, so that $\sigma_{v}\left(\tau_{2}\right) \in \widehat{S}$. This gives a diffeomorphism $\phi: \Sigma \rightarrow \widehat{S}$, and hence

$$
\text { vol } \widehat{S}=\int_{\widehat{S}} d V_{\widehat{S}}=\int_{\Sigma} \phi^{*}\left(d V_{\widehat{S}}\right)=\int_{\Sigma} J d V_{\Sigma}
$$

where $J=\operatorname{det} D \phi$ is the Jacobian of $\phi$. Since both $\widehat{S}$ and $\Sigma$ are space-like, the function $u$ is a Lipschitz function, (cf. [11]), whose (weak) derivative is uniformly bounded, since both normal vectors $N$ and $T$ lie in compact subsets of interior null cones. In addition, the (uniform) time $\tau$ exponential map, mapping $\Sigma$ to $\Sigma_{\tau}$ has Jacobian uniformly bounded above and below on $\left[-\frac{r_{2}}{2}, r_{2}\right]$, by the bound (2.7). (Recall that $H=\operatorname{tr} K$ measures the infinitesimal volume expansion or contraction). It follows that the Jacobian $J$ is uniformly bounded below, (depending only on $C, D$ ). Hence,

$$
\operatorname{vol} \Sigma \geq v_{1} \cdot \operatorname{vol} \widehat{S}
$$


Now the lower bound on $\operatorname{vol} B_{p}\left(\frac{1}{2}\right) \subset S$ in (1.9) does not immediately imply a lower bound on $\operatorname{vol} \widehat{S}$; (it could apriori happen that most all of the volume of $B_{p}\left(\frac{1}{2}\right)$ occurs outside $\widehat{S}$ ). However, in this case one can repeat all the estimates (2.6)-(2.9) when the construction of $\mathcal{C}$ is based at other center points $q^{\prime}$ in place of $q$. Thus, for $p^{\prime} \in B_{p}\left(\frac{1}{2}\right) \subset S$, define $q^{\prime}$ as in $(2.2)$ and let $\mathcal{C}^{\prime}$ be then as in (2.4). The same estimates as above then hold in $\mathcal{C}^{\prime}$. The corresponding domains $\widehat{S}^{\prime}=S \cap \mathcal{C}^{\prime}$ give a covering of $B_{p}\left(\frac{1}{2}\right) \subset S$. Hence the volume bound in (1.9) and the estimates above now do give the existence of points $p_{o} \in B_{p}\left(\frac{1}{2}\right) \subset S$ such that

$$
\operatorname{vol} \Sigma_{p_{o}} \geq v_{2}>0
$$

where $\Sigma_{p_{o}}$ is the level set of $\tau$, (i.e. $\tau_{o}$ ), containing $p_{o}$ and $v_{2}=v_{2}\left(v_{o}, D, C_{1}\right)$. (The local estimate (2.12) does not in fact depend on the absence of future cut points of $\exp _{q}$, cf. the discussion concerning (2.59) below).

Recall the standard volume comparison theorem in Riemannian geometry: if $(N, g)$ is a Riemannian $n$-manifold, with $\operatorname{Ric}_{g} \geq-(n-1) k$, then the ratio

$$
\frac{\operatorname{vol} D(r)}{\operatorname{vol} D_{k}(r)}
$$

is monotone non-increasing in $r$. Here $D(r)$ denotes the volume of a geodesic $r$-ball at any fixed point, while $D_{k}(r)$ is the geodesic $r$-ball in the $n$-dimensional space form of constant curvature $k$. It then follows from the curvature bound (2.9), together with (2.13), that the geodesic balls $D_{p_{o}}(r) \subset\left(\Sigma_{p_{o}}, g\right)$ satisfy

$$
\operatorname{vol} D_{p_{o}}(r) \geq v_{3} r^{n}
$$

for all $r \leq r_{2}$, where $v_{3}$ depends only on $v_{2}$ and $C_{3}$.

Observe that a similar estimate also holds for geodesic balls on other spatial slices $\Sigma_{\tau}$, with $\Sigma_{0}=\Sigma_{p_{o}}$, for $\tau \in\left[-\frac{r_{2}}{2}, r_{2}\right]$. Namely, the $L^{\infty}$ bound on $K$ in (2.7) bounds the infinitesimal distortion in the spatial metrics, and hence distances and volumes, under the flow of $N$. It follows that within the cylinder $\mathcal{C}_{o}$ centered at $p_{o}$, the volume estimate above holds for balls $D_{p_{\tau}}(r) \subset \Sigma_{\tau}$; thus, for $p_{\tau}=\sigma_{v_{o}}(\tau)$, where $p_{o}=\sigma_{v_{o}}(0)$, and for $r \leq r_{2}$, one has

$$
\operatorname{vol} p_{p_{\tau}}(r) \geq v_{4} r^{n}
$$

$v_{4}=v_{4}\left(v_{o}, C, D\right)$. An upper bound on the volume of $v o l D_{p_{\tau}}(r)$ of the form (2.14) follows immediately from the curvature bound (2.9).

In the construction above, we have shifted the original base point $p$ to a new base point $p_{o}$. However, one may now use these estimates to obtain equivalent volume bounds for the slices $\Sigma_{\tau}$ within the original cylinder $\mathcal{C}$ centered at $p$. This may be done by constructing a suitable chain, of bounded cardinality, of overlapping cylinders $\mathcal{C}_{i}$ from $\mathcal{C}_{o}$ to $\mathcal{C}$. One then uses the arguments above on each $\mathcal{C}_{i}$, together with the fact that upper and lower volume bounds of spatial slices are equivalent to upper and lower volume bounds of each cylinder $\mathcal{C}_{i}$.

Thus, in the following, we work on the original cylinder $\mathcal{C}$ from $(2.4)$ centered at $p$; the bound (2.14) holds with $p$ in place of $p_{o}$.

\section{Step III. (Local Coordinates).}

In this step, we define the cylinder $C_{r_{o}}$ and the local coordinate system on it, and obtain in addition some initial estimates on $\mathbf{g}_{\alpha \beta}$. The local coordinates are Gaussian in time and harmonic in space, (Gaussian-harmonic coordinate system).

Thus, the function $\tau$ from $(2.3)$ is chosen as the time coordinate on $\mathcal{C}$. To construct spatial harmonic coordinates, start with the slice $\Sigma=\Sigma_{0}$ within $\mathcal{C}$. By (2.9) and (2.14), one has the bounds

$$
\left|R_{g}\right| \leq C_{3}, \quad \operatorname{vol}_{g} D_{p}\left(r_{2}\right) \geq v_{4}
$$


It then follows, for instance from the discussion in $\S 1$, that there exists $r_{o}>0$, depending only on $C_{3}$ and $v_{4}$, such that the geodesic ball $D_{p}\left(r_{o}\right) \subset \Sigma$ admits a harmonic coordinate system $\left\{x_{i}\right\}$, $1 \leq i \leq n$, in which the spatial metric $g=\left.\mathbf{g}\right|_{\Sigma}$ is controlled in $L^{2, p}$, i.e.

$$
\left\|g_{i j}\right\|_{L^{2, p}} \leq R_{o}
$$

where the $L^{2, p}$ norm is taken on $D_{p}\left(r_{o}\right)$, and $R_{o}=R_{o}\left(C_{3}, v_{4}, p\right)$. The harmonic functions $x_{i}$ are solutions to the Dirichlet problem

$$
\Delta_{g} x_{i}=0,\left.\quad x_{i}\right|_{\partial D}=\phi_{i},
$$

where $D=D_{p}\left(r_{o}\right)$ and $\phi_{i}$ are suitably chosen boundary values, (approximating linear-type functions, cf. [12]).

Let $\phi_{i, \tau}=\phi_{i} \circ \psi_{\tau}$, where $\psi_{\tau}$ is the time $\tau$ flow from $\Sigma_{\tau}$ to $\Sigma_{0}$ along the integral curves of $N$. Thus, $\psi_{\tau}$ maps a domain $D_{\tau} \subset \Sigma_{\tau}$ diffeomorphically onto $D$ and $\phi_{i, \tau}$ are functions defined on $\partial D_{\tau}$. It follows that

$$
N\left(\phi_{i, \tau}\right)=1 \text { at } \partial D_{\tau} .
$$

Define the functions $x_{i}$ on $D_{\tau}$ to be solutions to the Dirichlet problem

$$
\Delta_{g_{\tau}} x_{i}=0,\left.\quad x_{i}\right|_{\partial D_{\tau}}=\phi_{i, \tau} .
$$

By (2.9) and (2.14), the estimate (2.15) holds uniformly on $D_{\tau}$, for $\tau \in\left[-\frac{r_{2}}{2}, r_{2}\right]$. Hence, as with $\Sigma_{0}, r_{o}>0$ may be chosen, depending only on $C_{3}$ and $v_{4}$, such that the functions $\left\{x_{i}\right\}$ form a harmonic coordinate system on $D_{\tau} \subset \Sigma_{\tau}$, on which one has the bounds

$$
\left\|g_{i j}\right\|_{L^{2, p}} \leq R_{o}
$$

where the $L^{2, p}$ norm is taken on $D_{\tau}$ and $g=g_{\tau}$. The estimate (2.20) holds for all $|\tau| \leq r_{o}$.

This construction gives the local coordinate system $\left(\tau, x_{i}\right), 1 \leq i \leq n$, on the $r_{o}$-cylinder

$$
C_{r_{o}}=D_{p}\left(r_{o}\right) \times\left[-r_{o}, r_{o}\right]
$$

about $p$, where the product structure is defined by the flow of $\nabla \tau$. For the remainder of the proof, $\Sigma_{\tau}$ is now redefined to be its intersection with $C_{r_{o}}$, i.e. $\Sigma_{\tau} \equiv D_{\tau}$.

The metric $\mathbf{g}$ in these coordinates has the form

$$
\mathbf{g}=\left(-1+|\xi|^{2}\right)(d \tau)^{2}+g_{i j}\left(d x_{i}+\xi_{i} d \tau\right)\left(d x_{j}+\xi_{j} d \tau\right),
$$

where $\xi=\left\{\xi_{i}\right\}$ is the shift vector. Thus,

$$
\partial / \partial \tau=N+\xi
$$

with $N=\nabla \tau$. The lapse function $\alpha$ of this foliation satisfies $\alpha \equiv 1$.

On each slice $\Sigma_{\tau} \subset C_{r_{o}}$, one has good spatial control, namely for $g_{i j}=\mathbf{g}_{i j}=\left.\mathbf{g}\right|_{\Sigma \tau},(2.20)$ holds. As usual, Latin indices $i, j$, denote spatial variables, i.e. $1 \leq i, j \leq n$, while Greek indices $\alpha, \beta$ denote space-time variables, $0 \leq \alpha, \beta \leq n$.

In the following, all Sobolev norms $L^{k, p}$ are understood to be spatial norms, i.e. the derivatives and norms are taken on spatial leaves $\Sigma_{\tau}$. Thus, for emphasis or clarity, we sometimes write $L_{x}^{k, p}$ in place of $L^{k, p}$. All estimates will be independent of $\tau$, for $\tau \leq r_{o}$.

Step IV. ( $L^{2, p}$ Estimates of $\left.g_{\alpha \beta}\right)$.

In this next step, we extend the estimate (2.20) to include the remaining terms $\mathbf{g}_{0 \alpha}, 0 \leq \alpha \leq n$, and also obtain estimates on the time derivatives of $\mathbf{g}_{\alpha \beta}$.

Before beginning, we first improve the estimate $(2.7)$ on the $2^{\text {nd }}$ fundamental form. Recall the Simons', (or Bochner-Weitzenbock) formula, cf. [3, Ch. 1I]:

$$
D^{*} D K=\delta d K+d \delta K-\mathcal{R}(K),
$$


on $\left(\Sigma_{\tau}, g_{\tau}\right)$, where the term $\mathcal{R}(K)$ is linear in the curvature and $K$; the exact form of $\mathcal{R}(K)$ plays no role in the argument, but for completeness is given by $\mathcal{R}(K)=R i c \circ K+K \circ R i c-2 R \circ K$, where $R \circ K$ is the action of the curvature tensor $R$ on symmetric bilinear forms. The elliptic operator $D^{*} D=-\operatorname{tr} D^{2}$ is the so-called rough Laplacian.

In the following, we frequently write $f \in L^{k, p}$ or $f \in L_{x}^{k, p}$ as shorthand for $f$ is uniformly bounded in $L^{k, p}$ along the spatial slices $\Sigma_{\tau},|\tau| \leq r_{o}$.

By (2.10), $d K \in L^{\infty}$, and hence $\delta d K \in L_{x}^{-1, p}$, for all $p<\infty$; recall that these spaces are defined as following (1.13). Similarly, by (2.11), since $d d=0$,

$$
d \delta K=d(\boldsymbol{\operatorname { R i c }} N) \in L_{x}^{-1, p}
$$

here we recall that the operators $\delta$ and $d$ are spatial. The term $\mathcal{R}(K)$ is also bounded in $L^{\infty}$. Hence, one has

$$
D^{*} D K=Q_{1},
$$

where $Q_{1}$ is uniformly bounded in $L_{x}^{-1, p}$, for any $p<\infty$, while $K$ is uniformly bounded in $L^{\infty}$. By (2.20), the coefficients of $D^{*} D$ in the local coordinates $\left\{x_{i}\right\}$ are controlled in $L^{2, p} \subset C^{1, \gamma}$. It then follows from standard elliptic regularity theory, cf. [10], that

$$
|K|_{L_{x}^{1, p}} \leq C_{2}
$$

where $C_{2}=C_{2}\left(C, v_{o}, c_{o}, D, p\right)$, on all spatial slices $\Sigma_{\tau},|\tau| \leq r_{o}$.

\section{Spatial Estimates.}

Here, we prove that the components $\mathbf{g}_{0 \alpha}$ also satisfy the $L^{2, p}=L_{x}^{2, p}$ estimate (2.20) uniformly on $\Sigma_{\tau}$. One has $N=\mathbf{g}^{0 \alpha} \partial_{\alpha}, \nabla x_{i}=\mathbf{g}^{i \alpha} \partial_{\alpha}$. Hence, $N\left(x_{i}\right)=\left\langle N, \nabla x_{i}\right\rangle=\mathbf{g}^{0 \alpha} \mathbf{g}^{i \beta} \mathbf{g}_{\alpha \beta}=\mathbf{g}^{0 i}$.

To obtain estimates on $N\left(x_{i}\right)$, differentiate the harmonic coordinate condition $(2.17)$, in the normal, (i.e. $N$ ), direction. Let $x_{i}^{\prime}=N\left(x_{i}\right)$. Since $\Delta x_{i}=0$, a standard computation, cf. $[3,1.184]$ for example, gives

$$
\Delta x_{i}^{\prime}=-\Delta^{\prime} x_{i}=\left\langle D^{2} x_{i}, K\right\rangle-\left\langle d x_{i}, \delta K+\frac{1}{2} d H\right\rangle .
$$

Here, as above and in the following, all metric quantities in (2.26) are on spatial slices $\Sigma_{\tau}$.

By $(2.25), K$ is uniformly bounded in $L^{1, p}$. The term $D^{2} x_{i}$ is also uniformly bounded in $L^{1, p}$, since by (2.20) the spatial metric is uniformly bounded in $L^{2, p}$ and hence the coordinate functions are uniformly bounded in $L^{3, p}$. Further, both $\delta K$ and $d H$ are uniformly bounded in $L^{p}$. Thus,

$$
\Delta x_{i}^{\prime}=Q_{2},
$$

where $Q_{2}$ is uniformly bounded in $L^{p},|\tau| \leq r_{o}$. As before, the coefficients of $\Delta$ are controlled in $L^{2, p}$. Further, by construction, cf. $(2.18), x_{i}^{\prime}=1$ on $\partial \Sigma_{\tau}$. Hence, standard elliptic regularity again gives

$$
\left\|x_{i}^{\prime}\right\|_{L^{2, p}}=\left\|\mathbf{g}^{0 i}\right\|_{L^{2, p}} \leq C_{4},
$$

where $C_{4}=C_{4}\left(C, v_{o}, c_{o}, D, p\right)$. Observe also that

$$
\mathrm{g}^{00}=-1,
$$

(since the lapse function $\alpha \equiv 1$ ). Hence $\mathbf{g}^{0 \alpha} \in L^{2, p}$, i.e. the $L^{2, p}$ norm of $\mathbf{g}^{0 \alpha}$ is uniformly bounded, $0 \leq \alpha \leq n$.

From this and (2.20), it is then an elementary exercise in linear algebra to see that

$$
\left\|\mathbf{g}_{\alpha \beta}\right\|_{L^{2, p}} \leq C_{5} \text {. }
$$

Briefly, $\mathbf{g}^{0 \gamma}=\left(\operatorname{det}_{\alpha \beta}\right)^{-1} A_{0 \gamma}$, where $A_{0 \gamma}$ is the $(0, \gamma)$ cofactor in the matrix $\mathbf{g}_{\alpha \beta}$. The cofactor $A_{00}$ involves only $g_{i j}$, and hence by $(2.20), A_{00} \in L^{2, p}$. Thus $\operatorname{det} \operatorname{g}_{\alpha \beta} \in L^{2, p}$. The same reasoning on $\mathbf{g}^{0 \alpha}$ then gives $A_{0 \alpha} \in L^{2, p}$, for all $\alpha$. Each determinant $A_{0 k}$ may be expanded along the first 
column to obtain a linear form in the variables $\mathbf{g}_{0 i}$, with coefficients $(n-1) \times(n-1)$ determinants. Thus, one has a linear system of $n$ equations in $n$ unknowns $\mathbf{g}_{0 i}$. The matrix of this system is the $(n-1)$-compound $G_{n-1}$ of the matrix $\left[g_{i j}\right]$, i.e. $\left(G_{n-1}\right)_{k l}=\operatorname{det} A_{k l}$, where $A_{k l}$ is the $(k, l)$ cofactor of $\left[g_{i j}\right]$. Since $\left[g_{i j}\right]$ is non-singular, and since $\left[g_{i j}\right]$ non-singular implies that $G_{n-1}$ is non-singular, (cf. $[8, \S 1.4]$ for instance), it follows that this linear system is invertible.

The components $\mathbf{g}_{0 i}$ are rational expressions in $\left\{g_{i j}\right\}$ and $\left\{A_{0 k}\right\}$, each of which is now bounded in $L^{2, p}$. Hence $\mathbf{g}_{0 i}$ is bounded in $L^{2, p}$. Finally, since $1=\mathbf{g}^{0 \alpha} \mathbf{g}_{\alpha 0}=\mathbf{g}^{00} \mathbf{g}_{00}+\mathbf{g}^{0 i} \mathbf{g}_{i 0}$, it follows from (2.28) that $\mathbf{g}_{00}$ is also bounded in $L^{2, p}$. This establishes the bound (2.29).

Recall that $\partial_{\tau}=N+\xi$, while $\left\langle N, \partial_{i}\right\rangle=0, i>0$, by construction. Since $\left\langle\partial_{i}, \partial_{\tau}\right\rangle=\mathbf{g}_{i 0}$ is bounded in $L^{2, p}$, it follows that $\left\langle\xi, \partial_{i}\right\rangle$ is bounded in $L^{2, p}$. Hence the shift vector $\xi=\left\{\xi_{i}\right\}$ is bounded in $L^{2, p}$,

$$
\|\xi\|_{L^{2, p}} \leq C_{6}
$$

This completes the $L^{2, p}$ estimates of $\mathbf{g}_{\alpha \beta}$ in spatial directions.

\section{$1^{\text {st }}$ Time Derivatives.}

Next we turn to estimates on the time derivatives of $\mathbf{g}_{\alpha \beta}$, i.e. $L^{1, p}$ estimates for $\partial_{\tau} \mathbf{g}_{\alpha \beta}$. To begin, using the Leibniz rule, and the fact that $\left[\partial_{\alpha}, \partial_{\tau}\right]=0$, it suffices to estimate

$$
\left\langle\nabla_{\partial_{\alpha}} \partial_{\tau}, \partial_{\beta}\right\rangle=\left\langle\nabla_{\partial_{\alpha}} N, \partial_{\beta}\right\rangle+\left\langle\nabla_{\partial_{\alpha}} \xi, \partial_{\beta}\right\rangle
$$

Suppose first $\alpha>0, \beta>0$, so $(\alpha, \beta)=(i, j)$. The first term in (2.31) is then $K_{i j}$, which is bounded in $L^{1, p}$ by (2.25), while the second term is also bounded in $L^{1, p}$ by $(2.30)$, (and the $L^{2, p}$ spatial bounds on $\mathbf{g}_{\alpha \beta}$ in $(2.29)$ ). This gives uniform $L^{1, p}$ bounds on $\partial_{\tau} g_{i j}$, i.e.

$$
\left\|\partial_{\tau} g_{i j}\right\|_{L_{x}^{1, p}} \leq C_{7}
$$

It follows of course that also

$$
\left\|\partial_{x_{k}} \partial_{\tau} g_{i j}\right\|_{L_{x}^{p}} \leq C_{7}
$$

The bounds on $\partial_{\tau} \mathbf{g}_{0 \alpha}$ require more work. Writing $\partial_{\tau}=N+\xi$ as above, the $L^{2, p}$ spatial estimates above imply that $\xi\left(\mathbf{g}_{0 \alpha}\right)$ is bounded in $L^{1, p}$, so one needs to obtain $L^{1, p}$ bounds on $N\left(\mathbf{g}_{0 \alpha}\right)$.

Recall that $x_{i}^{\prime}=N\left(x_{i}\right)=\mathbf{g}^{0 i}$. Hence $N\left(\mathbf{g}^{0 i}\right)=N N\left(x_{i}\right)=x_{i}^{\prime \prime}$. To obtain estimates on $x_{i}^{\prime \prime}$, differentiate the equation (2.17) in the $N$ direction twice. This gives

$$
\Delta x_{i}^{\prime \prime}=-\left(2 \Delta^{\prime} x_{i}^{\prime}+\Delta^{\prime \prime} x_{i}\right)
$$

Here, as before, all metric quantities are on the spatial slices $\Sigma_{\tau}$. It has already been proved that $x_{i}^{\prime} \in L^{2, p}$. From the form of $\Delta^{\prime}$ in $(2.26)$, one then easily sees that

$$
\Delta^{\prime} x_{i}^{\prime} \in L^{p}
$$

Next, one has

$$
\Delta^{\prime \prime} x_{i}=N\left\langle D^{2} x_{i}, K\right\rangle-N\left\langle d x_{i}, \delta K+\frac{1}{2} d H\right\rangle .
$$

To estimate these terms, let $e_{\alpha}$ be a local orthonormal basis on $\Sigma_{\tau}$, with $\nabla_{e_{\alpha}} e_{\beta}=0$ at any fixed point in $\Sigma_{\tau}$. Then the first term in (2.34) may be written

$$
N\left\langle D^{2} x_{i}, K\right\rangle=N\left(D^{2} x_{i}\left(e_{a}, e_{b}\right)\right) \cdot K\left(e_{a}, e_{b}\right)+D^{2} x_{i}\left(e_{a}, e_{b}\right) \cdot N\left(K\left(e_{a}, e_{b}\right)\right) .
$$

By (2.25) and (2.7), $K_{a b} \in L^{1, p}$ and $N K_{a b} \in L^{p}$, while by (2.20), $D^{2} x_{i} \in L^{1, p}$. Thus

$$
D^{2} x_{i}\left(e_{a}, e_{b}\right) \cdot N\left(K\left(e_{a}, e_{b}\right)\right) \in L^{p}
$$

Further $N\left(D^{2} x_{i}\left(e_{a}, e_{b}\right)\right)=N\left\langle\nabla_{e_{a}} d x_{i}, e_{b}\right\rangle=\left\langle\nabla_{N} \nabla_{e_{a}} d x_{i}, e_{b}\right\rangle$, so that

$$
N\left(D^{2} x_{i}\left(e_{a}, e_{b}\right)\right)=\left\langle\nabla_{e_{a}} \nabla_{N} d x_{i}, e_{b}\right\rangle+\left\langle R\left(N, e_{a}\right) d x_{i}, e_{b}\right\rangle-\left\langle\nabla_{e_{b}} d x_{i}, \nabla_{e_{a}} N\right\rangle .
$$


The curvature term in (2.36) is bounded in $L^{\infty}$, while the last term equals $\left\langle D^{2} x_{i}\left(e_{b}\right), K\left(e_{a}\right)\right\rangle$, which is bounded in $L^{\infty}$. For the first term, one has

$$
\left\langle\nabla_{e_{a}} \nabla_{N} d x_{i}, e_{b}\right\rangle=e_{a}\left\langle\nabla_{N} d x_{i}, e_{b}\right\rangle=e_{a}\left\langle\nabla_{e_{b}} d x_{i}, N\right\rangle=-e_{a}\left(K\left(d x_{i}, e_{b}\right)\right) .
$$

Since $K \in L^{1, p}$, this term is bounded in $L^{p}$. Combining these estimates, it follows that the first term in $(2.34)$ is bounded in $L^{p}$.

For the next term in (2.34), $N\left\langle d x_{i}, d H\right\rangle=\left\langle\nabla_{N} d x_{i}, d H\right\rangle+\left\langle d x_{i}, \nabla_{N} d H\right\rangle=\left\langle\nabla_{d H} d x_{i}, N\right\rangle+$ $\left\langle N, \nabla_{d x_{i}} d H\right\rangle$, so that

$$
N\left\langle d x_{i}, d H\right\rangle=-2 K\left(d H, d x_{i}\right),
$$

which is bounded in $L^{p}$.

Finally, $-N\left\langle d x_{i}, \delta K\right\rangle=N e_{a} K\left(d x_{i}, e_{a}\right)-N K\left(\nabla_{e_{a}} e_{a}, d x_{i}\right)-N K\left(e_{a}, \nabla_{e_{a}} d x_{i}\right)$. By (2.7), the latter two terms are in $L^{\infty}$. For the first term, write $N e_{a} K\left(d x_{i}, e_{a}\right)=e_{a} N K\left(d x_{i}, e_{a}\right)-\left(\nabla_{e_{a}} N\right)\left(K\left(d x_{i}, e_{b}\right)\right)$. The latter term here is $-\left[K\left(e_{a}\right)\right]\left(K\left(d x_{i}, e_{b}\right)\right)$, which is bounded in $L^{p}$ by $(2.25)$. For the first term, since $N K\left(d x_{i}, e_{a}\right)$ is bounded in $L^{\infty}, e_{a} N K\left(d x_{i}, e_{a}\right)=\operatorname{div}\left(N\left(K\left(d x_{i}\right)\right)\right)$ is bounded in $L^{-1, p}$, since the derivatives $e_{a}$ are spatial. This shows that

$$
N\left\langle d x_{i}, \delta K\right\rangle \in L^{-1, p} .
$$

Thus, combining these estimates on (2.33) gives a uniform bound on $\Delta x_{i}^{\prime \prime}$ in $L^{-1, p}$. On the boundary $\partial \Sigma_{\tau}$, one has $x_{i}^{\prime \prime}=0$. It then follows from elliptic regularity as before, (as in (2.24)), that

$$
N\left(\mathbf{g}^{0 i}\right)=x_{i}^{\prime \prime} \in L^{1, p} .
$$

Of course, by $(2.28), N\left(\mathbf{g}^{00}\right)=0$. As above, $\mathbf{g}_{0 i}$ is a rational expression in $\left\{g_{i j}\right\}$ and $\left\{A_{0 k}\right\}$. The bound (2.32) implies that each of these has $N$-derivative in $L^{1, p}$ and hence, by the same arguments as before, $N\left(\mathbf{g}_{0 \alpha}\right) \in L^{1, p}$. This gives uniform bounds

$$
\left\|\partial_{\tau} \mathbf{g}_{\alpha \beta}\right\|_{L_{x}^{1, p}} \leq C_{8}
$$

This completes the estimates for the first time derivatives on spatial slices. In particular, all Christoffel symbols are bounded in $L_{x}^{1, p}$.

$2^{\text {nd }}$ Time Derivatives.

Finally, we obtain $L^{p}$ estimates on the $2^{\text {nd }}$ time derivatives $\partial_{\tau} \partial_{\tau} \mathbf{g}_{\alpha \beta}$. To do this, take $\partial_{\tau}$ of the term $\left\langle\nabla_{\partial_{\alpha}} \partial_{\tau}, \partial_{\beta}\right\rangle$ in (2.31). One then obtains

$$
\left\langle\nabla_{\partial_{\alpha}} \nabla_{\partial_{\tau}} \partial_{\tau}, \partial_{\beta}\right\rangle+\left\langle\mathbf{R}\left(\partial_{\tau}, \partial_{\alpha}\right) \partial_{\tau}, \partial_{\beta}\right\rangle
$$

The curvature term is bounded in $L^{p}$, in fact $L^{\infty}$. (This uses the fact that $\xi$ is controlled, so the framing is controlled).

Write $\nabla_{\partial_{\tau}} \partial_{\tau}=\Gamma_{00}^{\gamma} \partial_{\gamma}$. Hence

$$
\left\langle\nabla_{\partial_{\alpha}} \nabla_{\partial_{\tau}} \partial_{\tau}, \partial_{\beta}\right\rangle=\Gamma_{00}^{\gamma}\left\langle\nabla_{\partial_{\alpha}} \partial_{\gamma}, \partial_{\beta}\right\rangle+\partial_{\alpha}\left(\Gamma_{00}^{\gamma}\right)\left\langle\partial_{\gamma}, \partial_{\beta}\right\rangle .
$$

By the first derivative estimates above, the Christoffel symbols are bounded in $L_{x}^{1, p}$. The first term is a product of Christoffel symbols, and hence is bounded in $L_{x}^{1, p / 2} \subset L^{n p /(2 n-p)}$, by Sobolev embedding. For $p>n$, (recall that $p$ is arbitrarily large), $L^{n p /(2 n-p)} \subset L^{p}$. Thus the first term is bounded in $L^{p}$.

For the second term, if $\alpha>0$, then $\partial_{\alpha}\left(\Gamma_{00}^{\gamma}\right)$ is bounded in $L^{p}$ by $(2.40)$. Hence, this gives

$$
\left\|\partial_{\tau} \partial_{\tau} g_{i j}\right\|_{L^{p}} \leq C_{9}
$$

uniformly in $\tau$. It remains to estimate the second time derivatives of $\mathbf{g}_{0 \alpha}$. These correspond to the second order time behavior of the shift vector $\xi$. 
These estimates are the most involved, and are the only estimates dependent on the Einstein equations. To obtain these estimates, one needs to differentiate (2.17) three times in the normal $N$ direction. Thus, from (2.17) again,

$$
\Delta x_{i}^{\prime \prime \prime}=-3 \Delta^{\prime} x_{i}^{\prime \prime}-3 \Delta^{\prime \prime} x_{i}^{\prime}-\Delta^{\prime \prime \prime} x_{i} .
$$

Recall $x_{i}^{\prime}=\mathbf{g}^{0 i}$ is bounded in $L^{2, p}$, while $x_{i}^{\prime \prime}=N\left(\mathbf{g}^{0 i}\right)$ is bounded in $L^{1, p}$. From previous work, it is then straightforward to bound the first two terms on the left in (2.42).

To see this, one has

$$
\Delta^{\prime} x_{i}^{\prime \prime}=\left\langle D^{2} x_{i}^{\prime \prime}, K\right\rangle-\left\langle d x_{i}^{\prime \prime}, \delta K+\frac{1}{2} d H\right\rangle,
$$

which is bounded in $L_{x}^{-1, p}$, since $x_{i}^{\prime \prime} \in L_{x}^{1, p}$. To estimate the term $\Delta^{\prime \prime} x_{i}^{\prime}$, one just replaces $x_{i}$ in the estimates (2.34)-(2.39) by $N\left(x_{i}\right)$. Using the fact that $N\left(x_{i}\right) \in L^{2, p}$, one sees by checking term by term that this is bounded in $L^{-1, p}$.

It remains to analyse

$$
\Delta^{\prime \prime \prime} x_{i}=N N\left\langle D^{2} x_{i}, K\right\rangle-N N\left\langle d x_{i}, \delta K+\frac{1}{2} d H\right\rangle .
$$

To begin,

$$
N N\left\langle D^{2} x_{i}, K\right\rangle=\left\langle\nabla_{N} \nabla_{N} D^{2} x_{i}, K\right\rangle+2\left\langle\nabla_{N} D^{2} x_{i}, \nabla_{N} K\right\rangle+\left\langle D^{2} x_{i}, \nabla_{N} \nabla_{N} K\right\rangle .
$$

By the Riccati equation (2.6), $\nabla_{N} K$ is bounded in $L^{\infty}$, while by the estimate on $(2.36), \nabla_{N} D^{2} x_{i}$ is bounded in $L^{p}$. Hence, the middle term in (2.44) is bounded in $L^{p}$. For the last term, taking the $N$-derivative of the Riccati equation (2.6) gives

$$
\left\langle D^{2} x_{i}, \nabla_{N} \nabla_{N} K\right\rangle=-2\left\langle D^{2} x_{i},\left(\nabla_{N} K\right) K\right\rangle-\left\langle D^{2} x_{i}, \nabla_{N} \mathbf{R}_{N}\right\rangle .
$$

The first term in (2.45) is bounded in $L^{\infty}$. The second (curvature) term in (2.45) will be analysed below.

For the first term in (2.44), one needs to take $N$-derivatives of all terms following (2.35) to (2.37). The only one which is not bounded in $L^{-1, p}$ by previous estimates is the term

$$
N\left\langle\mathbf{R}\left(N, e_{a}\right) d x_{i}, e_{b}\right\rangle=\left(\nabla_{N} \mathbf{R}\right)\left(N, e_{a}, d x_{i}, e_{b}\right)+\text { lower order. }
$$

We return again to this curvature term below, and proceed with the second term in (2.43).

Again, to estimate this, one takes $N$-derivatives of the estimates in (2.38) to (2.39). This gives first

$$
N K\left(d H, d x_{i}\right) \in L^{p}
$$

since $N K$ is bounded. For the $\delta K$ term, modulo lower order terms, this is of the form

$$
\begin{gathered}
N\left\langle\nabla_{N} \nabla_{e_{a}} K\left(d x_{i}\right), e_{a}\right\rangle=N\left\langle\nabla_{e_{a}} \nabla_{N} K\left(d x_{i}\right), e_{a}\right\rangle+N\left\langle\mathbf{R}\left(N, e_{a}\right) K\left(d x_{i}\right), e_{a}\right\rangle= \\
=\left\langle\nabla_{N} \nabla_{e_{a}} \nabla_{N} K\left(d x_{i}\right), e_{a}\right\rangle+\left(\nabla_{N} \mathbf{R}\right)\left(N, e_{a}, K\left(d x_{i}\right), e_{a}\right)= \\
=\left\langle\nabla_{e_{a}} \nabla_{N} \nabla_{N} K\left(d x_{i}\right), e_{a}\right\rangle+\left(\nabla_{N} \mathbf{R}\right)\left(N, e_{a}, K\left(d x_{i}\right), e_{a}\right)+\left\langle\mathbf{R}\left(N, e_{a}\right) \nabla_{N} K\left(d x_{i}\right), e_{a}\right\rangle ;
\end{gathered}
$$

the equalities here are understood to be modulo lower order terms. Modulo terms bounded in $L^{p}$, this may be rewritten as

$$
\nabla_{e_{a}}\left(\nabla_{N} \mathbf{R}\right)\left(N, K\left(d x_{i}\right), N, e_{a}\right)+\left(\nabla_{N} \mathbf{R}\right)\left(N, e_{a}, K\left(d x_{i}\right), e_{a}\right) .
$$

Combining these estimates gives then uniform $L_{x}^{-1, p}$ bounds on all terms in (2.43), except for the four curvature terms of the form $\left(\nabla_{N} \mathbf{R}\right) N$.

We obtain bounds on the curvature terms via the contracted $2^{\text {nd }}$ Bianchi identity on $(\mathbf{M}, \mathbf{g})$ :

$$
\delta \mathbf{R}=-\mathrm{dRic}
$$

or more precisely, cf. [3, 16.3],

$$
\boldsymbol{\delta} \mathbf{R}(X, Y, Z)=-\mathbf{d R i c}(Y, Z, X) .
$$


Write, on $\mathbf{M}$,

$$
\left(\nabla_{N} \mathbf{R}\right) N=-\boldsymbol{\delta} \mathbf{R}+\delta \mathbf{R}=\mathbf{d} \mathbf{R i c}+\delta \mathbf{R},
$$

where the divergences $\boldsymbol{\delta}, \delta$ are the space-time and space-like divergences on $\Sigma_{\tau}$ respectively.

Now the space-time curvature $\mathbf{R}$ is bounded in $L^{\infty}$, (in bounded framings). Hence the spatial divergence $\delta \mathbf{R}$ is bounded in $L_{x}^{-1, p}$, for any $p<\infty$. Thus, the curvature term (2.46) may be rewritten, modulo $L_{x}^{-1, p}$, as

$$
\left(\nabla_{N} \mathbf{R}\right)\left(N, e_{a}, d x_{i}, e_{b}\right)=\mathbf{d R i c}\left(d x_{i}, e_{b}, e_{a}\right) \in L_{x}^{-1, p},
$$

where the last estimate follows since all derivatives of Ric are taken in spatial directions. Similarly, for the second curvature term in (2.47), one has, for the same reasons, modulo $L_{x}^{-1, p}$,

$$
\left(\nabla_{N} \mathbf{R}\right)\left(N, e_{a}, K\left(d x_{i}\right), e_{a}\right)=\mathbf{d R i c}\left(K\left(d x_{i}\right), e_{b}, e_{a}\right) \in L_{x}^{-1, p} .
$$

This leaves left the two curvature terms:

$$
\left\langle D^{2} x_{i}, \nabla_{N} \mathbf{R}_{N}\right\rangle \text { and } \nabla_{e_{a}}\left(\nabla_{N} \mathbf{R}\right)\left(N, K\left(d x_{i}\right), N, e_{a}\right) .
$$

The second term is of form $\delta \mathbf{d R i c}\left(N, K\left(d x_{i}\right)\right.$ ), (to leading order), which cannot be controlled without the Einstein equation, since it involves differentiation in the $N$ direction. Similarly,

$$
\nabla_{N} \mathbf{R}_{N}=\operatorname{dRic}(N, \cdot, \cdot) \text {, modulo } L_{x}^{-1, p}
$$

is not controlled without the Einstein equations.

Dropping the usual constants, the Einstein equations on (M, g) are

$$
\text { Ric }-\frac{\mathbf{R}}{2} g=\mathcal{T},
$$

where $\mathcal{T}$ is the stress-energy tensor. Suppose ( $\mathbf{M}, \mathbf{g})$ is vacuum, or more generally, suppose the stress-energy tensor $\mathcal{T}$ satisfies

$$
d \mathcal{T}\left(N, e_{a}, e_{b}\right) \in L_{x}^{-1, p},
$$

where $e_{a}, e_{b}$ are spatial. Since the Einstein equations and the bound (2.5) imply that $\nabla_{e_{a}} \mathcal{T}(N) \in$ $L_{x}^{-1, p},(2.53)$ is equivalent to

$$
\nabla_{N} \mathcal{T} \in L_{x}^{-1, p}, \text { or } \mathcal{L}_{N} \mathcal{T} \in L_{x}^{-1, p},
$$

where $\mathcal{L}_{N}$ denotes the Lie derivative in the direction $N$.

Combining the bound (2.53) with the estimates obtained above on the terms in (2.42) then gives

$$
\Delta x_{i}^{\prime \prime \prime}=\Delta N N\left(\mathbf{g}^{0 i}\right) \in L_{x}^{-2, p},
$$

since $\delta \mathbf{d R i c}\left(N, K\left(d x_{i}\right)\right) \in L_{x}^{-2, p}$, not $L_{x}^{-1 . p}$. As before, since the coefficients of the Laplacian are well-controlled, (i.e. bounded in $L^{2, p}$ ), and the functions $x_{i}^{\prime \prime \prime}$ have 0 boundary values, elliptic regularity gives

$$
N N\left(\mathbf{g}^{0 i}\right) \in L^{p} .
$$

(One sees this from duality in the standard way, using the fact that $\Delta: L_{o}^{2, p} \rightarrow L^{p}$ is an isomorphism, cf. also [13]).

Applying the linear algebra argument as before then gives the bound

$$
\partial_{\tau} \partial_{\tau} \mathbf{g}_{0 \alpha} \in L^{p}
$$

This completes all of the estimates on $\mathbf{g}_{\alpha \beta}$. We refer to Remark 2.1 below for further discussion on use of the Einstein equations. Finally, the assumptions (2.53) or (2.54) are equivalent to (1.13). To see this, both $T$ and $N$ lie within a compact subset of $T^{+} \Omega$. Since the covariant derivative w.r.t. $T$ or $N$ involves only the pointwise behavior of these vector fields, one may replace $T$ by $N$ in (1.13), which then corresponds to (2.54). 
Step V. (Issue of Cut Points).

In this final step, we show that by passing to a smaller cylinder if necessary, of definite size within $C_{r_{o}}$, the exponential map $\exp _{q^{\prime}}$ at a suitable base point $q^{\prime}$, has no future cut points. By the work above, this will complete the proof.

To begin, return to the "cone" $\mathcal{C}$ in (2.4). Let $V$ be the collection of time-like unit vectors in $T_{q}^{+} \mathcal{D}_{r_{1}}$ for which there is a maximal geodesic $\sigma_{v}$ issueing from $q$ and terminating in $\mathcal{C}$. Let

$$
\widetilde{\mathcal{C}}=\left\{s v: v \in V, s \leq r_{1}\right\} \subset T_{q}^{+} \mathcal{D}_{r_{1}},
$$

so that in particular $\exp _{q} \widetilde{\mathcal{C}}$ contains $\mathcal{C}$. Here, as in Step I, $r_{1}$ is chosen so that no geodesic $\sigma_{v}(s)$, for $v \in V$, has conjugate points with $s \leq r_{1}$. Of course the geodesics $\sigma_{v}(s)$ now are no longer necessarily maximal. Since $\exp _{q}$ is of maximal rank on $\widetilde{\mathcal{C}}$, we work on the pullback $(\widetilde{\mathcal{C}}, \widetilde{\mathrm{g}})$, where

$$
\widetilde{\mathbf{g}}=\left(\exp _{q}\right)^{*} \mathbf{g} .
$$

The domain $\widetilde{\mathcal{C}}$ is a compact connected cone in $T_{q} \mathbf{M}$, w.r.t. the vector space structure. By the Gauss Lemma, the straightline generators of this cone are geodesics in the $\widetilde{\mathbf{g}}$ metric. Let $\widetilde{\tau}$ denote the distance to the origin $\{0\}$ w.r.t. $\widetilde{\mathrm{g}}$, within $\widetilde{\mathcal{C}}$. This is now a smooth function on $\widetilde{\mathcal{C}} \backslash\{0\}$, and serves as the parameter for the geodesics from $\{0\}$. Inside the cutlocus of $\exp _{q}$ on $\widetilde{\mathcal{C}}$, (i.e. where $\exp _{q}$ is a diffeomorphism), $\widetilde{\tau}$ is just the lift of the function $\tau$ from (2.3), up to an additive constant.

The level sets $\widetilde{\Sigma}_{\widetilde{\tau}} \subset \widetilde{\mathcal{C}}$ of $\tau$ are smooth, and hence the images $\exp _{q} \widetilde{\Sigma}_{\widetilde{\tau}}$ are smoothly immersed submanifolds in $\mathcal{C}$. The original Lipschitz level surface $\Sigma_{\tau}$ is just the part of $\exp _{q} \widetilde{\Sigma}_{\widetilde{\tau}}$ contained in the domain $U^{\tau}=\{x: \tau(x) \geq \tau\} \cap \mathcal{C}$. Observe that the timelike exponential map $\widetilde{\exp }_{0}$ of $(\widetilde{\mathcal{C}}, \widetilde{\mathbf{g}})$ based at 0 is a diffeomorphism onto $\widetilde{\mathcal{C}}$; this map has no conjugate or cut points within $\widetilde{\mathcal{C}}$.

Thus, we are now in exactly the same situation as at the end of Step I, with $(\widetilde{\mathcal{C}}, \widetilde{\mathbf{g}})$ in place of $(\mathcal{C}, \mathbf{g})$. Set $\widetilde{S}=\left(\exp _{q}\right)^{-1}(\mathcal{C}) \subset \widetilde{\mathcal{C}}$, so that $\widetilde{S}$ is an embedded hypersurface in $\widetilde{\mathcal{C}}$. Since $\exp _{q}$ is a local isometry of $\widetilde{S}$ onto $\widehat{S}$, one has

$$
\operatorname{vol}_{\widetilde{g}} \widetilde{S} \geq \operatorname{vol}_{g} \widehat{S}
$$

Hence, by the same reasoning as in Step II, the volume estimate (2.14) holds on the smooth hypersurfaces $\widetilde{\Sigma}_{\widetilde{\tau}}$.

We may thus apply the work in Steps II - IV to conclude that there is an $r_{o}>0$, depending only on $C, D, c_{o}, v_{o}$, and an $r_{o}$-cylinder $\widetilde{C}_{r_{o}} \subset \widetilde{\mathcal{C}}$, centered on $\widetilde{p}$, on which there are coordinates $\left(\widetilde{\tau}, \widetilde{x}_{i}\right)$ in which the metric $\widetilde{\mathbf{g}}$ is controlled in $L^{2, p}$ in the sense that (1.12) holds.

It remains to prove the existence of a suitable cylinder $C^{\prime}$ "downstairs", i.e. within $(\Omega, \mathbf{g})$, with these properties. Thus, let $\widetilde{\Sigma}_{\widetilde{\tau}}$ now denote the part of $\widetilde{\Sigma}_{\widetilde{\tau}}$ contained in $\widetilde{C}_{r_{o}}$. Each $\widetilde{\Sigma}_{\widetilde{\tau}}$ is an $n$-ball, topologically. Recall, as in Step II, that $\widetilde{S} \cap \widetilde{C}_{r_{o}}$ is a graph over $\widetilde{\Sigma}_{\widetilde{\tau}_{o}}$, for some $\widetilde{\tau}_{o}$. Hence, $\widetilde{S} \cap \widetilde{C}_{r_{o}}$ is also an $n$-ball topologically. By construction, both $\widetilde{\Sigma}_{\widetilde{\tau}_{o}}$ and $\widetilde{S} \cap \widetilde{C}_{r_{o}}$ have a uniform lower bound on their volume and size, i.e. $\operatorname{dist}_{\widetilde{g}}\left(\widetilde{p}, \partial\left(\widetilde{S} \cap \widetilde{C}_{r_{o}}\right)\right) \geq r_{3}>0$, (and similarly for $\left.\widetilde{\Sigma}_{\widetilde{\tau}_{o}}\right)$. These bounds depend only on the initial bounds on $C, v_{o}$ and $D, c_{o}$.

Let $U$ be the interior of the cutlocus of $\left.\exp _{q}\right|_{\tilde{\mathcal{C}}}$. The domain $U$ is starshaped w.r.t. the origin $\{0\}$ in $T_{q}^{+} \mathcal{D}_{r_{1}}$. Further, again by construction, $\widetilde{S} \cap \widetilde{C}_{r_{o}}$ is contained in the closure $\bar{U}$ of $U$. If $\left(\widetilde{S} \cap \widetilde{C}_{r_{o}}\right) \cap \partial U \neq \emptyset$, one may perturb it slightly, along the geodesic straight lines to $\{0\}$, to obtain an $n$-ball $\widetilde{S}^{\prime} \subset \widetilde{C}_{r_{o}}$ with $\widetilde{S}^{\prime} \subset U$. As before, the ball $\widetilde{S}^{\prime}$ has a definite lower bound on its volume and its size.

The exponential map exp $p_{q}$ now gives a diffeomorphism, in fact an isometry, from $\left(\widetilde{S}^{\prime}, \widetilde{g}\right)$ to $\left(S^{\prime}, g\right)$, $S^{\prime}=\exp _{q} \widetilde{S}^{\prime}$. Let $\mathcal{D}^{\prime}$ be the domain of dependence of $S^{\prime}$ in $(\mathbf{M}, \mathbf{g})$. This gives a globally hyperbolic region $\mathcal{D}^{\prime} \subset(\mathbf{M}, \mathbf{g})$, with a simply connected Cauchy surface of definite size and volume. The work 
of Steps I - IV may now be applied to this situation within (M, g) to produce a new cylinder $C^{\prime}$, centered at $p$, satisfying the bounds (1.12).

This completes the proof of Theorem 1.2.

It is an open question whether Theorem 1.2 holds without an assumption of the form (1.13), i.e. whether there exist coordinate systems in which (1.12) holds under only the bounds (1.9).

Remark 2.1. From the physical point of view, most stress-energy tensors $\mathcal{T}$ derive from matter fields satisfying a hyperbolic system of PDE, of $1^{\text {st }}$ or $2^{\text {nd }}$ order. In such a situation, these equations can frequently be used to interchange a time derivative $\nabla_{N} \mathcal{T}$ on spatial slices, with a spatial derivative $\nabla_{X} \mathcal{T}$, modulo lower order terms, e.g. $\left(\nabla_{N} \mathcal{T}\right)(X) \sim\left(\nabla_{X} \mathcal{T}\right)(N)$, modulo lower order terms. This is exactly the process used via the $2^{\text {nd }}$ Bianchi identity above. For instance, this is easily seen to be the case for electromagnetic fields, via use of the Maxwell equation $\mathbf{d F}=0$.

When the matter equations allow for such time-space replacement, modulo lower order terms, the condition (1.13) is of course not necessary in Theorem 1.2.

Next we turn to the proof of Corollary 1.3.

Let $(\mathbf{M}, \mathbf{g})$ be a weakly regular space-time, satisfying the size conditions, and satisfying the bound (1.9) distributionally. Thus, the components of $\mathbf{R}$, well-defined as distributions, are in fact bounded in $L^{\infty}$.

Any such space-time $(\mathbf{M}, \mathbf{g})$ is a limit of a sequence of $\left(C^{\infty}\right)$ smooth space-times $\left(\mathbf{M}, \mathbf{g}_{k}\right)$, cf. [9, Theorem 4] for instance. The metrics $\mathbf{g}_{k}$ are obtained in the usual way by taking the convolution of $\mathbf{g}$ with a sequence of smooth mollifiers. The local size conditions and local volume bound in (1.9), with $\delta_{o}$ in place of $\frac{1}{2}$, depend only on the $C^{0}$ behavior of the metric. Since the convergence to the limit is $C^{0}$, it follows that local size conditions and local volume bounds hold uniformly on the sequence $\left(\mathbf{M}, \mathbf{g}_{k}\right)$. Similarly, the fact that $|\mathbf{R}|_{T}$ is bounded on $\left(\mathbf{M}, \mathbf{g}_{k}\right)$ implies that the curvature $\left|\mathbf{R}_{\mathbf{g}_{k}}\right|_{T_{k}}$ of $\left(\mathbf{M}, \mathbf{g}_{k}\right)$ is uniformly bounded, for unit time-like vector fields $T_{k} \rightarrow T$, as $k \rightarrow \infty$.

It then follows from Theorem 1.2 that for any $p \in \Omega$, there are $r_{o}$-cylinders $\left(\left(C_{r_{o}}\right)_{k}, p_{k}\right) \subset \subset$ $\left(\mathbf{M}, \mathbf{g}_{k}\right)$, with $p_{k} \rightarrow p$, and coordinates on $\left(C_{r_{o}}\right)_{k}$ in which the metric $\mathbf{g}_{k}$ is controlled: this in the sense that the bounds (1.12) hold, with the exception of the bound on $\partial_{0} \partial_{0} \mathbf{g}_{0 \alpha}$. Since the bounds on $C, v_{o}$ and $D, c_{o}$, hold uniformly on $\left(\mathbf{M}, \mathbf{g}_{k}\right)$, and $p_{k}$ remains a bounded distance away from $\partial \mathbf{M}$, it follows that there is a limit cylinder $\left(C_{r_{o}}, p\right) \subset(\mathbf{M}, \mathbf{g})$ on which (1.12) holds, again except for the bound on $\partial_{0} \partial_{0} \mathbf{g}_{0 \alpha}$.

This proves the first part of Corollary 1.3. For the second part, one needs to make sense of the condition (1.13) on (M, g). It suffices to do this locally, i.e. on cylinders $C_{r_{o}} \subset(\mathbf{M}, \mathbf{g})$. Of course $\nabla_{N}$ Ric, or equivalently $\nabla_{N} \mathcal{T}$, is well-defined in $L^{-1, p}(\Omega)$, (since Ric $\in L^{\infty}$ ), but one needs to define it in $L^{-1, p}\left(\Sigma_{\tau}\right)$.

To do this, from the above, we know that $g_{\tau}=\left.\mathbf{g}\right|_{\Sigma_{\tau}}$ is in $L_{x}^{2, p}\left(\Sigma_{\tau}\right)$, for any $\Sigma_{\tau} \subset C_{r_{o}}$. Let $h$ be a symmetric bilinear form in $L^{1, q}\left(\Sigma_{\tau}\right)$, of compact support, for a given $\tau$. Extend $h$ into $C_{r_{o}}$ by the flow of $N$, so that $\mathcal{L}_{N} h=0$. Hence, $h$ is defined on all $\Sigma_{\tau} \subset C_{r_{o}}$. Formally, or alternately on the smooth approximations $\left(\mathbf{M}, \mathbf{g}_{k}\right)$ of $(\mathbf{M}, \mathbf{g})$, one has

$$
\int_{\Sigma_{\tau}}\left\langle h, \nabla_{N} \mathbf{R i c}\right\rangle d V=\int_{\Sigma_{\tau}} N\langle h, \mathbf{R i c}\rangle d V-\int_{\Sigma_{\tau}}\left\langle\nabla_{N} h, \mathbf{R i c}\right\rangle d V .
$$

Observe that $\nabla_{N} h=\mathcal{L}_{N} h+h\left(\nabla_{\partial_{a}} N, \partial_{b}\right)+h\left(\partial_{a}, \nabla_{\partial_{b}} N\right)=h\left(K\left(\partial_{a}\right), \partial_{b}\right)+h\left(K\left(\partial_{b}\right), \partial_{a}\right)$. Since $K \in L^{1, p}\left(\Sigma_{\tau}\right)$ and $p$ is large, it follows $\nabla_{N} h$ is well-defined in $L^{q}\left(\Sigma_{\tau}\right)$, for any $\tau$. Hence, the second term in (2.60) is well-defined, (since Ric is bounded in $L^{\infty}$ ).

For the first term on the right in (2.60), one has

$$
\int_{\Sigma_{\tau}} N\langle h, \mathbf{R i c}\rangle d V=\frac{d}{d \tau} \int_{\Sigma_{\tau}}\langle h, \mathbf{R i c}\rangle d V-\int_{\Sigma_{\tau}}\langle h, \mathbf{R i c}\rangle H d V .
$$


The second term in (2.61) is well-defined, since again $H \in L^{1, p} \subset C^{\alpha}$. Thus, to define (1.13) on $C_{r_{o}}$, we require that the derivative

$$
\frac{d}{d \tau} \int_{\Sigma_{\tau}}\langle h, \mathbf{R i c}\rangle d V, \text { or equivalently, } \frac{d}{d \tau} \int_{\Sigma_{\tau}}\langle h, \mathcal{T}\rangle d V
$$

exist for all $\tau \in\left[-r_{o}, r_{o}\right]$, for any $h$ as above. Under this condition, the bound (1.13) on $C_{r_{o}}$ is then equivalent to a uniform bound on (2.62), for all $\tau \in\left[-r_{o}, r_{o}\right]$.

Given this definition of the condition (1.13) on the limit $C_{r_{o}}$, it follows from the same proof as in Theorem 1.2 that $\partial_{0} \partial_{0} \mathbf{g}_{0 \alpha}$ is uniformly bounded in $L^{p}\left(\Sigma_{\tau}\right),|\tau| \leq r_{o}$. This completes the proof of Corollary 1.3.

\section{Concluding Remarks.}

We conclude the paper with several remarks extending the validity of Theorem 1.2, together with some open problems.

Theorem 1.2 gives the existence of cylinders $C$ of a definite size in the interior of $\mathbf{M}$, and a definite distance away from any boundary $\partial \mathbf{M}$, on which there exist coordinates in which the metric is controlled in $L^{2, p}$. Of course, by rescaling up suitably to realize the size conditions, applying Theorem 1.2, and then rescaling back down, the cylinders $C$ may be chosen to be arbitrarily close to $\partial \mathbf{M}$. (This is already implicit in Corollary 1.3). However, the coordinates may change in the (smaller and smaller) cylinders as one approaches $\partial \mathbf{M}$.

The main reason it is necessary to stay a definite distance away from $\partial \mathbf{M}$ in the proof is that one needs to work in globally hyperbolic regions, as in (2.1), which are future 1-connected. If $\mathbf{M}$ is globally hyperbolic and future 1-connected to begin with, then it is no longer necessary to stay a given distance away from $\partial \mathbf{M}$. We describe an alternate version of Theorem 1.2 in this context.

Thus, suppose (M, g) is globally hyperbolic and future 1-connected; (for instance, ( $\mathbf{M}, \mathbf{g}$ ) has a simply connected Cauchy surface). Choose any point $q \in \mathbf{M}$ and time-like unit vector $N_{o} \in T_{q}^{+} \mathbf{M}$. Let $V \subset T_{q}^{+} \mathbf{M}$ be the compact cone of unit vectors forming a fixed angle $\theta$ with $N_{o}$, and let $\mathcal{C}=\exp _{q} \tau v, v \in V$, be the corresponding "cone" in (M, g). Also, let $N$ be the corresponding vector field tangent to the geodesics $\sigma_{v}(\tau)=\exp _{q}(\tau v)$, and let $\Sigma_{\tau}$ be the level sets of $\tau$ in $\mathcal{C}$. We make the following assumptions: there exist constants $C<\infty$ and $v_{o}>0$ such that

$$
\begin{gathered}
|\mathbf{R}|_{N} \leq C, \text { within } \mathcal{C}, \\
\operatorname{vol} \Sigma_{\tau} \geq v_{o} \cdot\left(\operatorname{diam} \Sigma_{\tau}\right)^{n} .
\end{gathered}
$$

Although (3.2) is understood to hold for all $\tau \leq r_{1}$, for a fixed $r_{1}$, the methods used in Step II show that it suffices to assume (3.2) holds for a fixed $\tau_{o}>0$, where the size $\tau_{o}$ depends only on $C$ in (3.1). The bound (3.2) is of course scale-invariant.

We then have the following result, valid at least up to a point in $\partial \mathbf{M}$.

Corollary 3.1. Let (M, g) be a globally hyperbolic, future 1-connected space-time satisfying the assumptions (3.1)-(3.2). Suppose also (M, g) is vacuum, or more generally, satisfies (1.13). Then there exist small constants $d_{o}>0$, and $r_{o}>0$, depending only on $C, v_{o}, N_{o}$ and $\theta$, such that if

$$
\sigma_{N_{o}}(\tau) \in \mathbf{M}, \forall \tau<d_{o}
$$

then there exist coordinates $\left(\tau, x_{i}\right)$ on the cylinder

$$
C_{r_{o}}\left(d_{1}\right)=D_{p}\left(r_{o} d_{o}\right) \times\left[\frac{d_{1}}{2}, d_{1}\right] \subset \mathbf{M},
$$

in which the metric satisfies the bounds (1.12). Here $d_{1}$ is the largest value such that $d_{1} \leq d_{o}$ and $C_{r_{o}}\left(d_{1}\right)$ is contained in $\mathbf{M}$. 
The proof of this result is exactly the same as that of Theorem 1.2. In fact it is simpler, since the issues of global hyperbolicity and future 1-connected are assumed, and one works with $N$ in place of the vector field $T$ from Definition 1.1. Thus, for $d_{o}$ small as above, and for $C(V)$ the cone on $V$ in $T_{q} \mathbf{M}$, the exponential map $\exp _{q}$ restricted to $(C(V) \backslash\{0\}) \cap B_{0}\left(d_{1}\right)$ is a diffeomorphism onto $\left(\mathcal{C} \cap B_{q}\left(d_{1}\right)\right) \backslash q$, where $B_{q}\left(d_{1}\right)=\exp _{q}\left(B_{0}\left(d_{1}\right)\right)$. The domain $\mathcal{C} \cap B_{q}\left(d_{1}\right)$ plays exactly the same role as $\mathcal{C}$ in Step I. All the estimates of Steps II-IV then proceed just as before and are uniform for $\tau \in\left[\frac{d_{1}}{2}, d_{1}\right]$.

Remark 3.2. One may also derive a version of Theorem 1.2, (or Corollary 3.1), without the lower volume bound in (1.9). Thus, one has a uniform spatial curvature bound (2.9), either on the slices $\Sigma_{\tau} \subset \mathcal{C}$ downstairs, or on the slices $\widetilde{\Sigma}_{\widetilde{\tau}} \subset \widetilde{\mathcal{C}}$ upstairs in $T_{q} \mathbf{M}$. (The bound (2.9) does not require any apriori volume bound). If the volume $\Sigma_{\tau}$ or $\widetilde{\Sigma}_{\widetilde{\tau}}$ is very small, then the injectivity radius of $g_{\tau}$ is very small, i.e. the spatial metric $g_{\tau}$ is highly collapsed in the sense of Cheeger-Gromov. However, (as before), the Rauch comparison theorem implies that the intrinsic exponential map on $\Sigma_{\tau}$ or $\widetilde{\Sigma}_{\widetilde{\tau}}$ is still of maximal rank on geodesic balls of a definite size, (depending only on the curvature bound). Suppose first one works in the situation of $\Sigma_{\tau} \subset \mathcal{C}$. Then just as before in time-like directions, one can lift the metric $g_{\tau}$ up to the tangent space by pulling back by its exponential map, i.e. consider the metric $\widetilde{g}_{\tau}=\left(\exp _{p_{\tau}}\right)^{*} g_{\tau}$ defined on balls in $T_{p_{\tau}} \Sigma_{\tau}$. The metric $\widetilde{g}_{\tau}$ has a uniform lower bound on the volumes of small balls. All the arguments in Steps II-IV can then be carried out as before on this "unwrapped" space-time and the corresponding unwrapped cylinder $\widetilde{C}_{r_{o}}$. This gives coordinates $\left(\widetilde{\tau}, \widetilde{x}_{i}\right)$ for $\widetilde{C}_{r_{o}}$ on which the metric $\widetilde{\mathbf{g}}$ satisfies the bounds (1.12). The same procedure holds when working with $\widetilde{\Sigma}_{\widetilde{\tau}}$. These coordinates upstairs within $T_{q} \mathbf{M}$ give then "multi-valued" coordinates downstairs in $(\mathbf{M}, \mathbf{g})$.

To conclude, we mention two open problems. First, for certain purposes, the $L^{\infty}$ bound on the curvature in (1.11) may be viewed as too strong. It would be of interest to know if a version of Theorem 1.2 holds with suitable $L^{p}$ bounds on $|\mathbf{R}|_{T}, p>n / 2$, in place of $L^{\infty}$ bounds.

Second, it would be very interesting if the Einstein equations could be used to remove the dependence of these results on bounds on the full curvature, i.e. if bounds on the full curvature could be replaced by bounds on the Ricci curvature, possibly introducing other hypotheses not related to curvature. This seems to be a challenging problem; cf. [1] for some further discussion.

\section{REFERENCES}

[1] Anderson, M.: Cheeger-Gromov theory and applications to general relativity, Proc. 2002 Cargèse School on General Relativity, (to appear), gr-qc/0208079.

[2] Beem, J, Ehrlich, P and Easley K.: Global Lorentzian Geometry, $2^{\text {nd }}$ Edition, Marcel Dekker, New York, (1996).

[3] Besse, A.: Einstein Manifolds, Ergebnisse der Mathematik Series, 3:10, Springer Verlag, Berlin, (1987).

[4] Clarke, C.J.S.: Singularities in globally hyperbolic space-time, Comm. Math. Phys., 41, (1975), 65-78.

[5] Clarke, C.J.S.: Local extensions in singular space-times II, Comm. Math. Phys., 84, (1982), 329-331.

[6] Clarke, C.J.S.: The Analysis of Space-Time Singularities, Cambridge Lecture Notes in Physics, 1, Cambridge Univ. Press, London, (1993).

[7] Ellis, G.F.R. and Schmidt, B: Singular space-times, Gen. Rel. and Gravitation, 8, (1977), 915-953.

[8] Gantmacher, F.: The Theory of Matrices, vol. 1, Chelsea Publishing Co, New York, (1960).

[9] Geroch, R. and Traschen, J.: Strings and other distributional sources in general relativity, Phys. Review D36, (1987), 1017-1031.

[10] Gilbarg, D. and Trudinger, N.: Elliptic Partial Differential Equations of Second Order, $2^{\text {nd }}$ Edition, Springer Verlag, Berlin, (1983).

[11] Hawking, S.W. and Ellis, G.F.R.: The Large Scale Structure of Space-Time, Cambridge Univ. Press, London, (1973).

[12] Jost, J. and Karcher, H.: Geometrische Methoden zur Gewinnung von a-priori Schranken für harmonische Abbildungen, Manuscripta Math., 40, (1982), 21-71. 
[13] Lions, J.L. and Magenes, E.: Non-Homogeneous Boundary Value Problems and Applications, I, Grundlehren Series, 130, Springer Verlag, Berlin, (1966).

[14] Tipler, F., Clarke, C.J.S. and Ellis, G.F.R: Singularities and horizons: a review article, in General Relativity and Gravitation, vol. 2, A. Held, (Ed.), Plenum Press, New York, (1980), 87-206.

September, 2002

Department of Mathematics

S.U.N.Y. at Stony Brook

Stony Brook, N.Y. 11794-3651

anderson@math.sunsyb.edu 